\title{
FRACTAL DIMENSIONS FROM CHAOTIC OSCILLATIONS IN SEMI-INSULATING GaAs
}

\author{
A. ZDUNIAK AND J. LUSAKOWSKI \\ Institute of Experimental Physics, University of Warsaw \\ Hoża 69, 00-681 Warszawa, Poland
}

\begin{abstract}
Relaxation and domain current oscillations in undoped semi-insulating $\mathrm{GaAs}$ were observed at room temperature for a broad range of voltage applied to a sample. The oscillations were characterized by a reconstruction of an attractor of the system. An analysis of the attractor helped to discriminate between the two types of oscillations. A transition from one type of oscillations to the other was connected with a chaotization of the current. A chaotic state of the system was analyzed by calculations of fractal dimensions $D_{q}$ for $-0.6<q<40$ and the $f(\alpha)$ function.
\end{abstract}

PACS numbers: $05.45 .+\mathrm{b}, 72.20 . \mathrm{IIt}$

\section{Introduction}

Semi-insulating properties of undoped GaAs crystals are caused by a deep midgap level EL2 [1]. A room-temperature current-voltage $(I-V)$ characteristic of such a material is composed of three parts: for lowest electric fields one observes an ohmic behavior which is followed by a sublinear part and then by a region of a negative differential conductivity (NDC) $[2,3]$. Two types of current oscillations can be observed in this material. The first one are high electric field domain oscillations [3-5] and the other are relaxation oscillations [6-8]. A mechanism of the domain oscillations can be found in Ref. [5] and that of the relaxation oscillations in Ref. [7].

There are two factors that help to discriminate between the two types of the oscillations. The first one is the amplitude which is greater for the domain oscillations by a factor of about 50 . The other one is the shape of the oscillations which is sinusoidal for the relaxation oscillations and shows sharp peaks in the domain case. Stable relaxation oscillations are separated from stable domain oscillations by a transition region which is characterized by a strong chaotization of oscillations.

In the present paper another method of discrimination between the two types of oscillations is proposed. It is based on an analysis of attractors reconstructed from experimental oscillatory signals. A fractal analysis of an attractor of a chaotic state of oscillations is also presented. 


\section{Experiment}

Domain and relaxation oscillations were observed in several samples of undoped semi-insulating GaAs. The results obtained on one of them are described below. The sample used was a $4 \times 1 \times 0.4 \mathrm{~mm}^{3}$ slab supplied with two bar-shaped $\mathrm{Au}$ contacts. Stable relaxation oscillations were observed for the sample bias greater than $212 \mathrm{~V}$ and stable domain oscillations for the bias above $370 \mathrm{~V}$. A chaotic signal was observed for voltages between $310 \mathrm{~V}$ and $350 \mathrm{~V}$. The oscillations were characterized by a reconstruction of two-dimensional attractor for $240 \mathrm{~V}, 310 \mathrm{~V}$, $320 \mathrm{~V}$ and $400 \mathrm{~V}$. In each case the reconstruction was based on a 30000-point time series and a method of Grassberger and Procaccia [9] was used. A time delay used in the reconstruction was chosen as the first minimum of the mutual information of the signal [10]. Chaotic oscillations were characterized by a fractal analysis of an attractor which involved calculation of a spectrum of fractal dimensions $D_{q}$ for $-0.6<q<40$ and the $f(\alpha)$ function [11].

\section{Results}

Figure 1 shows reconstructions of an attractor of the investigated system for different types of oscillations. The attractor for stable relaxation (a) and stable
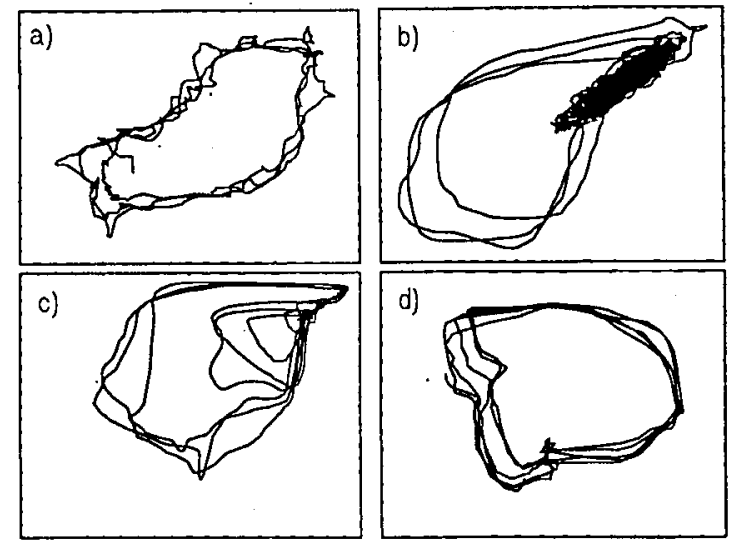

Fig. 1. Two-dimensional reconstruction of an attractor for stable relaxation (a), weakly chaotic (b), chaotic (c) and stable domain (d) oscillations. The measurements were performed at $305.5 \mathrm{~K}$ for a voltage applied to the sample equal to $240 \mathrm{~V}, 310 \mathrm{~V}, 320 \mathrm{~V}$ and $400 \mathrm{~V}$, respectively.

domain oscillations (d) has a shape of a cyclic orbit which reflects periodicity of the oscillations. Figure 1b shows an attractor of relaxation oscillations (black area) on which bumps of domain oscillations are superimposed. This clearly demonstrates that these are two different types of oscillations. Figure 1c corresponds to chaotic 
oscillations. One can notice that the structure of this attractor reflects sensibility of the system to initial conditions: two trajectories which are close in the upper right corner of the picture may appear divergent in another part of the phase space. This is just what lies behind a chaotic dynamics.

Figure 2 shows results of a numerical analysis of the attractor of the chaotic state. In Fig. 2a a spectrum of dimensions $D_{q}$ of the attractor is shown for
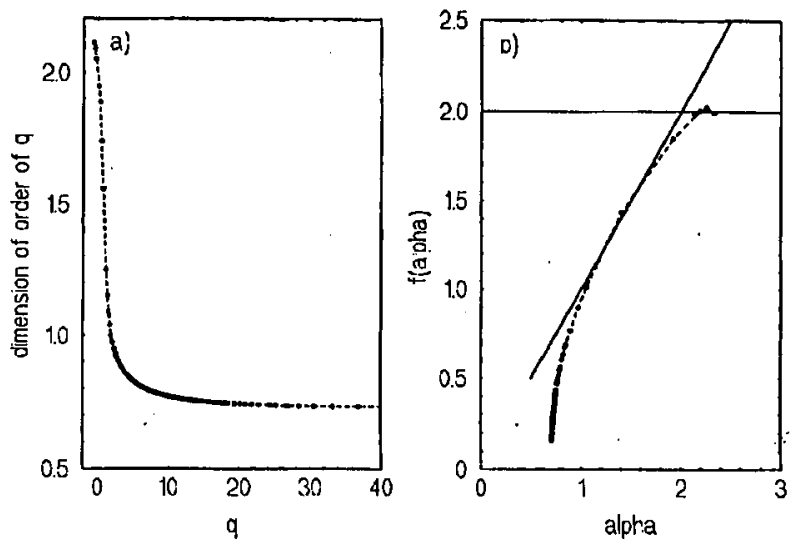

Fig. 2. The spectrum of fractal dimensions $D_{q}(\mathrm{a})$ and the $f(\alpha)$ function (b) for the attractor shown in Fig. 1c.

$-0.6<q<40$. The value of $D_{0}$ is equal to 2 which means that the attractor fills the embedding phase space. Thus, one can suspect that the topological dimension of the attractor is greater than 2 and a reconstrution in a higher-dimensional phase space is required. The shape of the $f(\alpha)$ function reveals a multifractal nature of the chaotic attractor, which gives origin to a broad range of values of the scaling index $\alpha$. It was verified that this range is much narrower for the $f(\alpha)$ function of an attractor of stable relaxation oscillations. The shape of the function follows theoretical predictions [11]: it is tangent to the $f(\alpha)=\alpha$ line and the value at the maximum is equal to $D_{0}$.

In conclusion, two types of current oscillations in semi-insulating GaAs were observed at room temperature and were discriminated by an analysis of an attractor of the system. A fractal nature of a chaotic attractor was also analyzed.

\section{Acknowledgments}

The authors are thankful to Dr. E. Kamińska and Dr. A. Piotrowska for preparing contacts to the sample and to Prof. M. Grynberg for discussions.

\section{References}

[1] M. Kamińska, Rev. Phys. Appl. 23, 793 (1988).

[2] A. Zduniak, J. Lusakowski, Actà Phys. P. ol. A 82, 777 (1992). 
[3] U. Rau, J. Peinke, J. Parisi, K. Karpierz, J. Eusakowski, W. Knap, Phys. Lett. A 157, 356 (1991).

[4] D.C. Northrop, P.R. Thornton, K.E. Trezise, Solid State Electron. 7, 17 (1964).

[5] D.A. Johnson, R.A. Puechner, G.N. Maracas, J. Appl. Phys. 67, 300 (1989).

[6] J. Eusakowski, Mater. Sci. Eng. B 6, 1 (1990).

[7] J. Eusakowski, A. Zduniak, submitted to publication.

[8] B. Willing, J.K. Maan, to be published in Proc. EPS Conference, Regensburg 1993.

[9] P. Grassberger, I. Procaccia, Physica D 9, 189 (1983).

[10] A.M. Fraser, H.L. Swiney, Phys. Rev. A 33, 1134 (1986).

[11] T.C. Halsey, M.H. Jensen, L.P. Kadanoff, I. Procaccia, B. Shraiman, Phys. Rev. A 33, 1141 (1986). 